\title{
Vitamin D functions as a shield in our immune system
}

\section{Introduction}

The term "Rheumatology" originates from the Greek term "revma" ("current"), a derivative of the verb "reo" ("circulate") which designates a movement towards a direction. The Greek physician Hippocrates was the first to use the term "rheumatism" for diseases related to the joints. He believed that the human organism is composed of four fluid substances: blood, phlegm, yellow bile and black bile. A movement disorder of one of these four fluid substances leads to diseases related to the joints and diseases in general. ${ }^{1}$

Approximately $2.500,000$ Greeks are estimated to be affected by a rheumatic disease nowadays, giving grounds to the belief that it occurs as an epidemic. More precisely, as far as the rheumatic diseases are concerned, he believed that indisposition and diseases are caused by a substance that begins in the brain and gets diffused like a "river" to all the parts of the body. ${ }^{1}$ A common feature of all the rheumatic diseases is the presence of an inflammation at the joints or another part of the musculoskeletal system or other organs as well that may lead to a chronic inflammation to a person and therefore a lesion at the tissues and organs where the inflammatory responses occur.

Inflammation is the response of the body's immune system to the harmful substances such as germs, viruses, chemical substances, injuries that invade our organism. The immune system is a complex network of cells and chemicals. Its natural role is to protect the organism and face any eventual infections. In case a disorder of this function of the immune system occurs for any reason whatsoever, the latter erroneously attacks the organs of the body itself, producing antibodies that fight off the cells of similar organs or vital tissues of the body. This state is known as "autoimmune disorder"; the so-called "autoimmune diseases". Proper nutrition at every age boosts the immune system, whereas poor nutrition results in its disorganization. In order to understand how important nutrition is for the development of the human immune system one has only to bear in mind that babies are born with an immune system at a "zero" point, this is why they are kept at a completely protected environment. The immune system development and maturity will be obtained through the food they consume, which during the first months is nothing but the mother's breast milk or formula milk (enriched in nutritional substances) for mothers who are unable to breastfeed their children while they are gradually introduced in the infant's diet. The same phenomenon appears at all stages of human life. Being an important fat-soluble vitamin, it is widely known as it helps the calcium absorption, expresses in a selective way the expression of the immune system cells and constrains the risk of autoimmune diseases such as diabetes type 1 , multiple sclerosis and rheumatic arthritis.

Vitamin D deficiency is related to many chronic diseases, as well as skeletal diseases, rickets, osteopathy, osteoporosis, the Crohn's disease. Despite the fact that the sun offers an advantage to Greek people since Vitamin D owes its availability to direct sunlight, recent researches demonstrate that we are confronted with a vitamin D deficiency. Among the most evident causes is the dark complexion,
Volume 6 Issue 2 - 2017

\author{
loanna Vasilakopoulou \\ Dietician - Nutritionist, Greece
}

Correspondence: loanna Vasilakopoulou, Dietician Nutritionist, Scientific coordinator of the scientific group "LOGO DIATROFIS", Founder member of the Greek Nutrition Society, Greece, Email info@gaiasdiet.gr

Received: January 27, 2017| Published: February 15, 2017

a quite common characteristic of Greek people while the people with darker complexion show a 3-7 times greater deficiency and the fact that we spend more time indoors, especially during the summertime. The annual fluctuations of the vitamin D levels, give its decline during the winter and the increase during the summertime demonstrated a negative correlation at least with the recurrence of rheumatic arthritis and the systemic lupus erythematosus. ${ }^{2}$

Nevertheless, studies show the power of vitamin D in preventing and treating diseases such as scleroderma, thyroiditis, hashimoto, psoriasis, uveitis, Reiter's syndrome. The improvement of the vitamin D uptake should be a priority in case of patients with autoimmune diseases and their relatives as well. ${ }^{2}$ The main dietary sources of vitamin D include fish oils, cod liver oil, fatty fish, herring, salmon, small fish where one can eat the bones as well, liver, egg yolks, butter, milk, cheese -especially the hard ones- ("kasseri", "graviera") and the enriched food such as soft margarine, juices, breakfast cereals. ${ }^{2,3}$

To sum up, it is vital to change our nutritional habits, following a balance diet which is enriched in fishes, dairy products, fruits and vegetables which are based on the famous Mediterranean diet. Moreover, keeping the weight stable is important to stay healthy and outdoor activities like walking a friend, hiking, or more traditional activities like swimming, tennis and biking in accessible areas of your country will help people as well. The main idea is to have a good time and stay active while keeping your vitamin D levels sufficient.

\section{Acknowledgements}

None.

\section{Conflict of interest}

The author declares no conflict of interest.

\section{References}

1. Hippocrates. The treatise On Ancient Medicine. Paris: E Littre; 1839. $1: 1-3$.

2. Dimitris Grigorakis. Autoimmune diseases, immune system \& nutrition. Greece; 2016.

3. Chandra RK. Nutrition and the immune system: an introduction. $A m L$ Clin Nutr. 1997;66(2):460S-463S. 